\title{
Cardiac Response to Live Music Performance: Computing Techniques for Feature Extraction and Analysis
}

\author{
Elaine Chew ${ }^{1}$, Peter Taggart ${ }^{2}$, Pier Lambiase ${ }^{2}$ \\ ${ }^{1}$ CNRS - UMR9912 / STMS (IRCAM), Paris, France \\ ${ }^{2}$ Institute for Cardiovascular Science, University College London, London, UK \\ ${ }^{3}$ Barts Heart Centre, London, UK
}

\begin{abstract}
Strong emotions and mental stress have been linked to potentially deadly arrhythmias. Music evokes strong emotion through the regulation of tension and release and the modulation of changes and transitions. We exploit this in a novel study involving patients with implanted cardiac defibrillators to study the impact of live music performance on cardiac electrophysiology. The patients' heart rates are artificially fixed with pacing at the higher of 80 beats per minute or 10 above the heart's intrinsic rate. We make continuous recordings directly from the heart muscle whilst the patients are listening to a short classical music concert, which is concurrently recorded in a separate stream. The participants provide annotations of perceived boundaries/transitions and felt tension. The recorded cardiac and music information is further processed to extract relevant features. Here, we describe the experiment design, and the mathematical and computing techniques used to represent and abstract the features from the recorded data. Cardiac reaction is measured by the action potential duration (APD), approximated using the action recovery interval (ARI). The expressive parameters extracted from the music include the time varying loudness, tempo, and harmonic tension. The synchronized information layers allow for detailed analysis of immediate cardiac response to dynamically varying expressive nuances in performed music.
\end{abstract}

\section{Introduction}

Strong emotions and mental stress have been linked to abnormal heart rhythms that in some people can be dangerous. However, the mechanisms by which emotions destabilize heart electrical activity and cause these abnormal heart rhythms are not well understood. Music induces strong emotions especially at moments of change or transition through violating or fulfilling expectations. These strong emotions can sometimes be associated with tension (stress) or pleasure. Here, we monitor cardiac changes during live music performance, comparing them to moments of musical change or transitions, to better understand the interactions between emotion responses and heart rhythm.

In the current study, patients fitted with intracardiac devices-biventricular pacemakers or biventricular ICDs-are invited to a short classical concert featuring music covering a range of moods and containing many changes. Data about participants' musical sophistication is collected. Participants' heart rates are artifically fixed with pacing at 80 beats per minute (bpm) or 10 above intrinsic. While participants listen to the music, electrical signals detected by their pacemaker or ICD from the surface of their heart is downloaded for analysis. Participants also rate their tension (stress) levels through recall, as well as perceived moments of change or transition in the music. We collect the signals and extract salient features so as to analyze changes to the patients' electrical heart signals and compare them to changes and transitions in the music.

This article is based, in part, on our IRAS ${ }^{1}$ application no. 242471, approved by the South Central - Oxford C Research Ethics Committee of the UK Health Research Authorities, with preliminary data demonstrations and results.

\subsection{Background}

Strong emotions and mental stress have been linked to deadly ventricular arrhythmias [ [1 2] $]$. However, the mechanisms by which emotions destabilize cardiac electrophysiology and cause ventricular arrhythmias are not well understood. Prior studies by the co-authors have demonstrated direct electrophysiological effects of mental stress on the heart [3, 4]. Using movie clips, statistically significant decreases were observed to the mean action potential duration (APD) in both the left and right ventricles between high stress mid-movie and end-movie sequences, and the control (matched breathing without movie). There

1 www.hra.nhs.uk/about-us/committees-and-services/ integrated-research-application-system 
was also statistically significant shortening of the APD between the low-stress movie-start sequence and the highstress mid-movie and end-movie sequences. While related studies using music exist [5-8], none have explored APD response, nor considered ecological music sources.

Our aim here is to study the impact of music, in particular music in ecological live performance situations and the emotions and stresses it induces, on cardiac electrophysiology. The goal is to correlate analytical measurements of expressive parameters such as tempo, loudness, and harmonic tension with listeners' physiological responses to identify musical signatures that provoke anxiety as well as promote pleasure, and chart their effects on cardiac electrophysiology so as to increase the understanding of the pathways that affect mood and heart rhythm.

\section{Study Design}

This section gives the rationale for the study design.

\subsection{The Concert}

For ecological validity, our study was designed to take place in a live concert setting. Each of the three concerts was attended by two-to-three patients to ensure device programmer and cardiologist coverage. Prior studies mentioned above focussed only on recorded music stimuli, either short duration audio of acoustic music or synthesized MIDI files. Studies have shown that emotion responses to music are heightened in situations of live performance [9], our choice for maximal cardiac response.

The music stimuli have also been chosen to exhibit sharp (and gradual) changes in musical properties, and to range widely in tension. The first concert featured Chopin's Ballade No.2, a romantic piano work, and music based on rhythms transcribed from ECG recordings of transitions in/out of Ventricular Tachycardia (VT) during an electrophysiology (EP) procedure. The latter were collaged from the Chopin Ballade and Mars from Holst's The Planets, which had similar rhythms, and are potentially stressful to the patients for non-musical reasons. Jonathan Berger's "Intermezzo," a modern piece, and Wilhelm Kempff's piano arrangement of the "Siciliano" from Bach's flute sonata (BWV 1031) were added to subsequent concerts.

\subsection{Heart Rhythm Acquisition}

Prior to each performance, participants' pacemakers or ICDs are programmed from CRT to dual chamber pacing and fixed at the higher of $80 \mathrm{bpm}$ or 10 above the heart's intrinsic rate. Pacing slightly above resting heart rates is essential because the APD is strongly affected by heart rate, decreasing with increasing heart rate and vice versa.
Patients are given ten minutes to adjust to the new setting while comfortably seated. Pacing, audio, and video are synchronized with claps. Pacing is maintained for the duration of the performance, then reverted to the original settings. Intracardiac electrogram (EGM) signals at $512 \mathrm{~Hz}$ resolution are downloaded from the pacemaker or ICD lead connected to the left ventricle (LV Distal tip 1) whilst participants are listening to the music.

\subsection{Music Annotations}

After each concert, participants provide information on the moments of change-abrupt alterations of acoustic properties or music structure - and transition — changes that take place over a period of time-that they perceived in the music. Audio and video recordings of the concert they just witnessed are played back to the participants during the annotation session to assist in recall. Boundary annotation is done with only audio feedback. Next, participants rate the tension felt during the performance-for the first concert, emotion annotations were also collected. The annotations were collected via existing (ELAN ${ }^{2}$ ) and bespoke software. Participants also complete a reduced Goldsmiths Musical Sophistication Index (Gold-MSI ${ }^{3}$ ) survey to assess their musical background.

\section{Computational Methods}

This section describes how the APD and musical parameters are extracted from the EGM and music audio signals.

\subsection{EGM: Action Potential Duration}

Cardiac reaction is measured by the APD, an electrical parameter of major importance in the development of serious and fatal rhythm disturbances. As in [4], the APD is approximated using the ARI computed from the EGM signal, $p$. The start of each segment is located using the

${ }^{2}$ tla.mpi.nl/tools/tla-tools/elan
3 www.gold.ac.uk/music-mind-brain/gold-msi

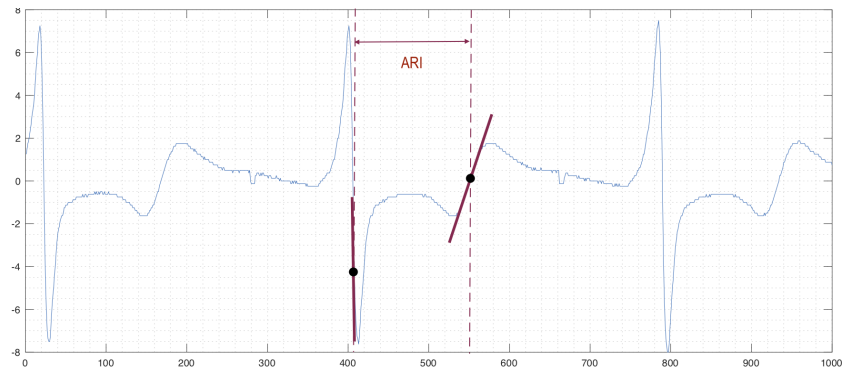

Figure 1. Activation recovery interval: plot shows the EGM signal, $p$, vs. frames $(512 \mathrm{~Hz})$ 
findpeaks function in MATLAB with suitable thresholds. The EGM signal from start to end of the altered pacing is then smoothed using loess regression (second-degree polynomial, span 20) to give $\tilde{p}$. A difference function then estimates the derivative, $d p / d t$, from the smoothed EGM signal. Inside a given segment, the ARI is given by the time between $\arg \max _{t} d p / d t$ and $\arg \min _{t} d p / d t$ as shown in Figure 1. The second plot in Figure 2 shows ARI values with a three-point weighted moving average smoothing. Normal probablity plots (not displayed) show that the ARI values follow an approximate normal distribution. Highlighted in the plot are data points more than 1.96 standard deviations from the mean. The graph shows that, for this patient, there are many more statistically significant long ARIs before the concert; and, more statistically significant shorter ARIs (associated with stress) after the concert.

\subsection{Audio: Loudness and Tempo}

Loudness is one of the most primal features in communication through music and sound. It is a perceptual quality that changes with frequency (same intensity sounds of different pitch have different loudness), duration (sensing loudness takes time, and transient loudness can be modeled by the peak while sustained loudness by the average) and masking by sounds in the same frequency band. We extract loudness values (in sones) from the recorded audio using the method outlined in [10], which uses the MATLAB music analysis (MA) toolbox 4 An example output can be seen in the third plot in Figure 2. Note that the cluster of statistically significant low ARI values around 840s and 860 s coincides with the first loud section in the Chopin.

Tempo tells us the rate at which the music is progressing from beat to beat, where beats are pulses one might tap to while listening to the music. Even state-of-the-art music beat trackers frequently fail when applied to music with highly variable beats, as is the case here, so the performer provides the beats (in seconds), $\left[b_{i}\right]$, by marking them manually on the recorded audio signal using SonicVisualiser 5 The instantaneous tempo (in beats per minute), $\left[v_{i}\right]$, is then $60 /\left(b_{i+1}-b_{i}\right)$ at time $t_{i}=\left(b_{i+1}-b_{i}\right) / 2$. The bottom plot in Figure 2 gives the performed tempo for Chopin, and the before/during/after VT rhythms of the Arrhythmia Suite, approximately the RR intervals of the ECGs from which the pieces were derived. Note that the arousing loud section with the low ARIs in the Chopin is also a fast one.

\subsection{Score: Harmonic Tension}

Musical tension is an essential part of music. Musicians choreograph expectation to evoke strong emotions. Expectation leads to anticipation; anticipation induces stress

4 www.pampalk.at/ma

5 sonicvisualiser.org
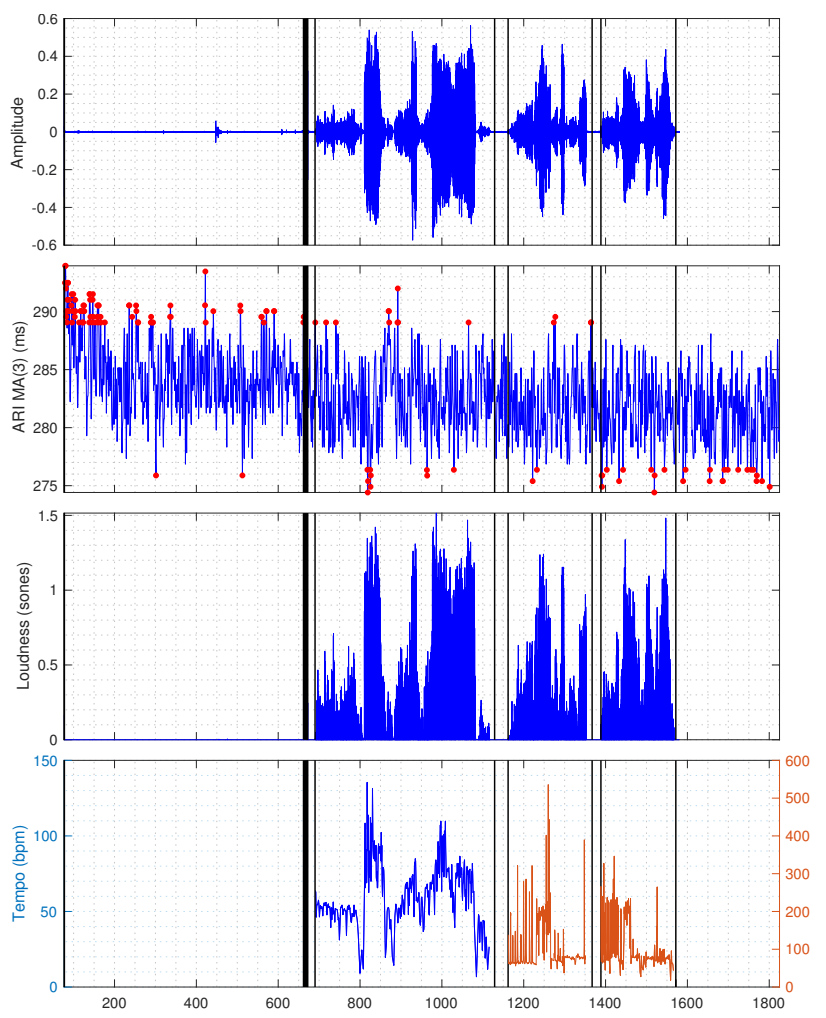

Figure 2. Action recovery intervals (second from top) computed from the EGM, aligned with the music audio signal (top) and audio loudness and music tempo/rhythm information; vertical lines delineate boundaries between the Chopin Ballade and the two Arrhythmia Suite pieces

and dopamine release [11]. The realization of the expected outcomes can trigger tension release, activating pleasure channels associated with food, drugs, and sex [12].

While tension can stem from a number of sources, including silence, the focus here is on harmonic tension. We use the method described in [13, 14] based on the spiral array [15] to compute three measures of harmonic tension: (1) cloud diameter (degree of dissonance or clashing sounds); (2) cloud momentum (how much/quickly the harmony changes); and, (3) tensile strain (how far the context has veered from the global key). Figure 3 shows the harmonic tension parameters aligned with the audio and ARI.

The lowest ARI value, $274.4 \mathrm{~ms}$ at $818.6 \mathrm{~s}$, marking the greatest stress response, occurs in the first instance of the stormy passage in the Chopin. It is not only loud and fast, it is also the moment of great harmonic instability, marked by high and rising cloud diameter/momentum and tensile strain. The highest ARI value during the concert, 292.0 $\mathrm{ms}$ at $892.2 \mathrm{~s}$, occurs in the languid, slow section after the storm, at the long-awaited harmonic resolution (release) back to the global key, with low diameter and tensile strain. 

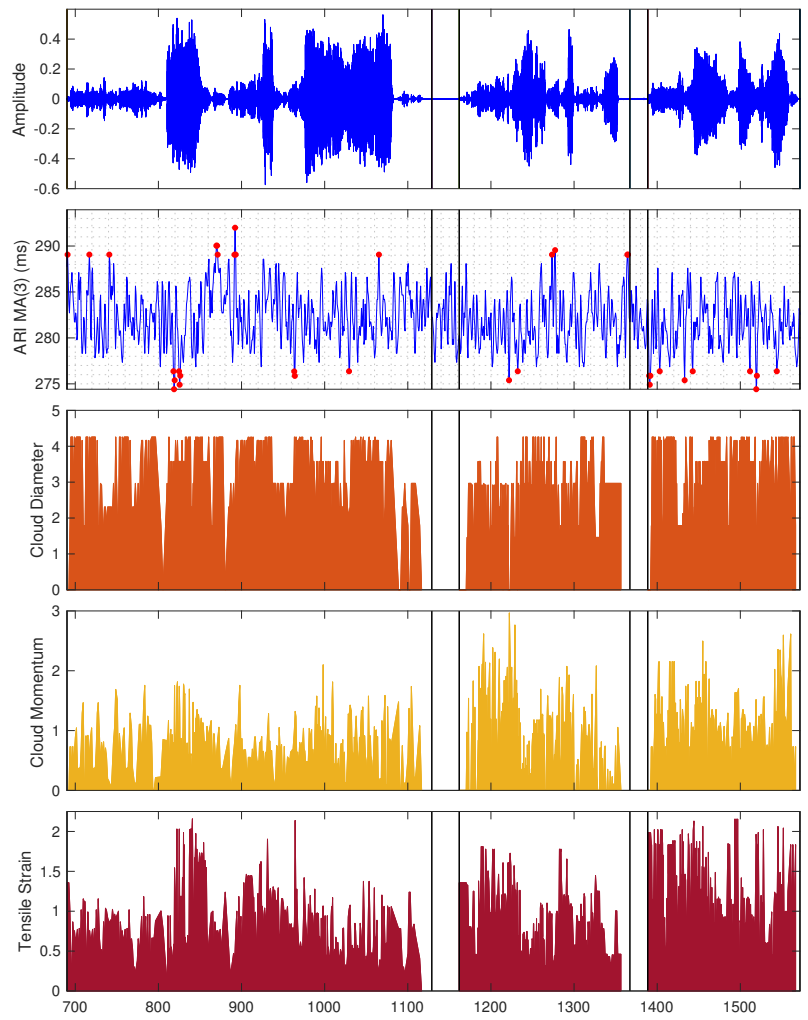

Figure 3. Action recovery intervals, aligned with the music audio (top), and three harmonic tension measures (bottom): dissonance (cloud diameter), chord change (cloud momentum), distance from global key (tensile strain)

\section{Future Work}

We have described the design and methodology of a research project that explores the connections between music and electric activity of the heart with the aim to understand the pathways that affect mood and heart rhythm. The initial prognosis is promising. By synchronizing and comparing the evolving APD to time-varying expressive parameters (loudness, tempo, and harmonic tension) in the music, we can detect instantaneous stress (and relaxation) responses and link them to developments in the music. Future analyses will reveal individual and group response patterns to specific structures in the live music performance.

\section{Acknowledgments}

We are grateful to Abbott, Barts Health NHS Trust, and members of the Music, Performance and Expressivity Lab for their support in realising this study. This research is funded, in part, by the European Research Council (ERC) under the European Union's Horizon 2020 Research and Innovation programme (Grant agreement No.788960).

\section{References}

[1] Lampert R. Mental stress and ventricular arrhythmias. Curr Cardiol Rep 2016;18(12):118.

[2] Lampert R. Behavioral influences on cardiac arrhythmias. Trends Cardiovasc Med 2016;26(1):68-77.

[3] Taggart P, Boyett M, Logantha S, Lambiase P. Anger, emotion, and arrhythmias. Front Physiol 2011;2(Article 67).

[4] Child N, Hanson B, Bishop M, Rinaldi C, Bostock J, Western D, Cooklin M, O’Neil M, Wright M, Razavi R, Gill J, Taggart P. Effect of mental challenge induced by movie clips on action potential duration in normal human subjects independent of heart rate. Circ Arrhythm Electrophysiol 2014;7(3):518-523.

[5] Bernardi L, Porta C, Sleight P. Cardiovascular, cerebrovascular, and respiratory changes induced by different types of music in musicians and non-musicians: the importance of silence. Heart 2006;92:445-452.

[6] Krabs R, Enk R, Teich N, Koelsch S. Autonomic effects of music in health and crohn's disease: The impact of isochronicity, emotional valence, and tempo. PLoS One 2015;10(5):e0126224.

[7] Cerruto G, Mainardi L, Koelsch S, Orini M. The periodic repolarization dynamics index identifies changes in ventricular repolarization oscillations associated with musicinduced emotions. In Proc. Intl Conf on Comp in Cardiology, volume 44. Rennes, France, 2017; 4 pages.

[8] Bretherton B, Deuchars J, Windsor W. The effects of controlled tempo manipulations on cardiovascular autonomic function. Music Science 2019;2:1-14.

[9] Coutinho E, Scherer K. The effect of context and audiovisual modality on emotions elicited by a musical performance. Psychol Music 2017;45(4):550-569.

[10] Kosta K, Ramirez R, Bandtlow O, Chew E. Mapping between dynamic markings and performed loudness: A machine learning approach. J Maths and Music 2016;(2):149172. Special Issue on Music and Machine Learning.

[11] Salimpoor V, Benovoy M, Larcher K, Dagher A, Zatorre R. Anatomically distinct dopamine release during anticipation and experience of peak emotion to music. Nature Neuroscience 2015;14(2):257-264.

[12] Mallik A, Chanda M, Levitin D. Anhedonia to music and to mu-opioids: Evidence from the administration of naltrexone. Nature Scientific Reports 2017;7:Article 41952.

[13] Herremans D, Chew E. Tension ribbons: Quantifying and visualising tonal tension. In Proc. Intl Conf on Tech for Music Notation \& Rep. Cambridge, UK, 2016; 8-18.

[14] Herremans D, Chew E. Morpheus: Generating structured music with constrained patterns and tension. IEEE Trans on Affective Computing 2017 (Early Access);16 pages.

[15] Chew E. Mathematical and Computational Modeling of Tonality. New York: Springer, 2014.

Address for correspondence:

Elaine Chew

IRCAM, 1 place Igor-Stravinsky, 75004 Paris, France

elaine.chew@ircam.fr 